\title{
Ovarian primary or secondary: cracking the nut
}

\section{Elamurugan Sujindra*, Sankappa P. Sinhasan, Sai Chandran Sabita, Arounassalame Bupathy}

Department of Obstetrics and Gynecology, Indira Gandhi Medical College and Research Institute, Puducherry, India

Received: 16 August 2016

Accepted: 14 September 2016

\author{
*Correspondence: \\ Dr. Sujindra Elamurugan, \\ E-mail: sujindra@ rediffmail.com
}

Copyright: (C) the author(s), publisher and licensee Medip Academy. This is an open-access article distributed under the terms of the Creative Commons Attribution Non-Commercial License, which permits unrestricted non-commercial use, distribution, and reproduction in any medium, provided the original work is properly cited.

\begin{abstract}
Ovarian cancer is one of the commonest gynecological cancers and ranks among the top five deadliest cancers in most countries. Tumors of the ovary are majority of the time primary with a small percentage of secondary malignancy. Metastasize to the ovaries can occur from many organs, including the stomach, small intestine, colon, rectum, gall bladder, appendix, pancreas, breast, uterus, fallopian tube, and peritoneum. Stomach is the primary site in $70 \%$ of cases. Carcinomas of colon, appendix, and breast are the next most common primary sites. These are referred to as Krukenberg tumor, accounting for $1 \%$ to $2 \%$ of all ovarian tumors. We report a case of 40 year old female presenting with bilateral solid and cystic ovarian tumor with small primary tumor located in sigmoid colon. The case report highlights the importance of preoperative workup needed to search for primary tumor, as Krukenberg's tumors are known for poor outcome.
\end{abstract}

Keywords: Krukenberg tumor, Adenocarcinoma, Sigmoid colon

\section{INTRODUCTION}

The incidence of cancer in India has been increasing recently due to various demographic, socioeconomic and cultural changes such as increased longevity, delayed childbearing, decreased parity and a more westernized lifestyle. ${ }^{1}$ Among these ovarian cancer is one of the commonest gynaecological cancers and ranks among the top five deadliest cancers in most countries. ${ }^{2}$

Tumors of the ovary are majority of the time primary with a small percentage of secondary malignancy. Metastasize to the ovaries can occur from many organs, including the stomach, small intestine, colon, rectum, gall bladder, appendix, pancreas, breast, uterus, fallopian tube, and peritoneum. Stomach is the primary site in most

Krukenberg tumor cases (70\%). Carcinomas of colon, appendix, and breast are the next most common primary sites. ${ }^{2}$ In our case report, we present one another atypical manifestation of Krukenberg tumor and how the patient was managed. The case presented here illustrates the difficulties encountered in the clinical and histopathological diagnosis of secondary over primary.

\section{CASE REPORT}

A 40 year old multiparous woman presented with complaints of abdominal distension for a period of three months. She also had associated history of dyspnea due to the distension of abdomen. She had no history of dyspepsia, weight loss or bleeding per rectum. There was no disturbance in bowel and bladder movements. Her medical and surgical history were unremarkable, except for that she has had three cesarean sections in the past and underwent tubectomy. Her menstrual history was normal. On physical examination, the patient appeared moderately built and nourished, not anemic. On examination of the abdomen it was found to be distended with presence of ascitis. Two large mobile masses of size approximately 12 and $10 \mathrm{~cm}$ were palpable in the right and left lower abdomen, respectively. Bimanual examination was done and the mass was confirmed to be of ovarian origin, mobile, floating in the ascitic fluid. The uterus was normal in size, mobile; pouch of Douglas was 
free of nodules. Per rectal examination was normal. An ultrasound of the abdomen was done which revealed bilateral ovarian masses. Right ovarian mass, measured $12 \times 10 \times 8 \mathrm{~cm}$ with predominantly solid and few cystic areas. Left ovarian mass measured $10 \times 8 \times 8 \mathrm{~cm}$ with predominantly cystic and few solid areas. Massive ascitis was noted. Uterus and other abdominal organs appeared normal. Serum CA-125 levels were $59 \mathrm{U} / \mathrm{mL}$ (normal range $\leq 35 \mathrm{U} / \mathrm{mL}$ ). Mantoux test was negative. Ascitic fluid cytology was negative for malignant cells. During her period of stay in the hospital for pre-operative evaluation, the patient developed massive ascitis with dyspnoea which was rapidly re-accumulating even on repeated tapping. Patient had ascitic fluid tapped four times (1.5-2 litters each time) prior to surgery. Three days prior to surgery, patient developed pleural effusion, which was also tapped (1.2 litters in two sittings). With all these findings, an initial diagnosis of ovarian cancer was made and patient was taken up for explorative laparotomy.

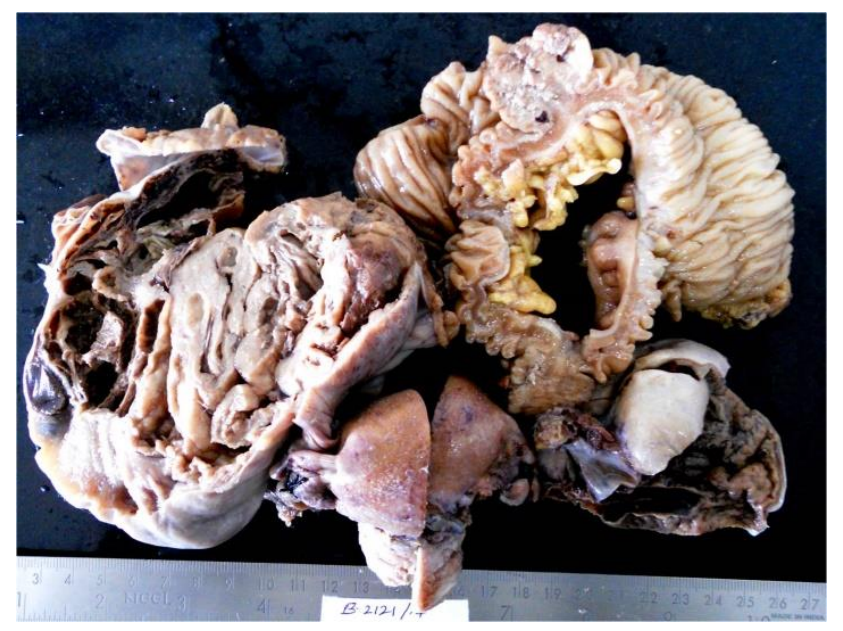

Figure 1: Gross specimen showing bilateral ovarian solid and cystic tumor masses with mucoid areas admixed with areas of hemorrhage and necrosis.

Resected segment of intestine is also seen.

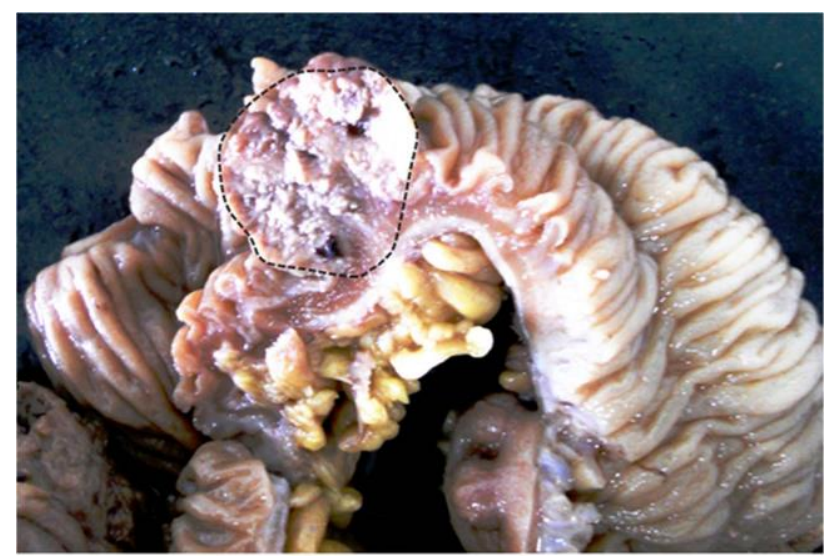

Figure 2: Resected Sigmoid colon showing intraluminal polypoidal mass measuring $2 \times 2 \mathrm{~cm}$.
At surgery, a midline vertical incision was made, and the abdominal cavity was opened. About 2 litters of hemorrhagic ascitic fluid were present and fluid sample was taken for cytology. The pelvis was completely filled with the ovarian masses on both sides. There was a breach in ovarian capsule on the right side. Uterus was normal in size, but the serosal layer showed tumor nodules. There were no adhesions. The patient underwent exploratory laparotomy with total abdominal hysterectomy and bilateral salpingo-ovariotomy and omentectomy. Subsequently, the abdomen was explored. There was a $2 \mathrm{~cm}$ umbilicated tumor nodule on the surface of the left lobe of liver. The diaphragm, stomach and small intestine were normal. Gross examination of the large intestine revealed a tumor mass in the sigmoid colon. Hence it was decided intra operatively to proceed with resection of intestinal segment containing tumor mass and end to end anastomosis. Cut open specimen of the sigmoid colon revealed a $3 \mathrm{~cm}$ intraluminal mass.

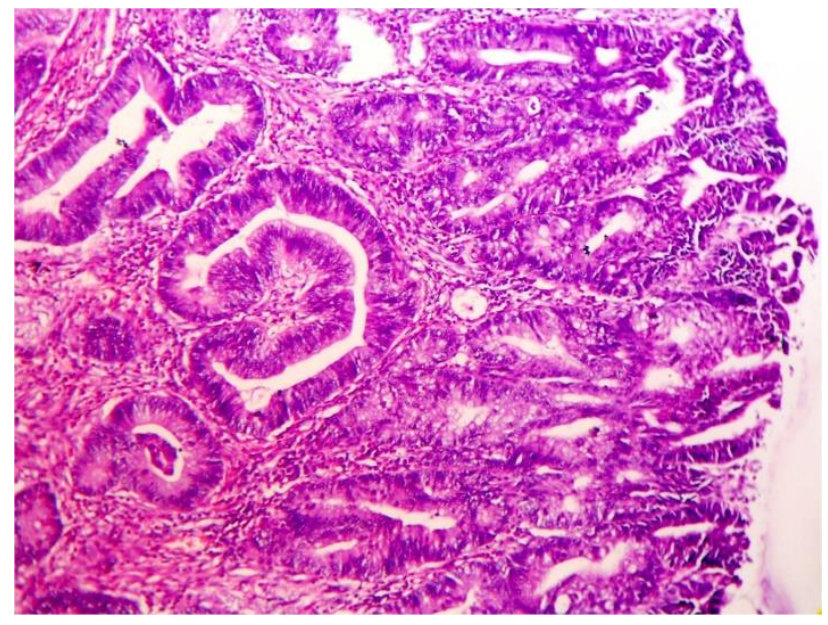

Figure 3: Histopathology section from sigmoid colon mass revealed adenoma-carcinoma sequence with well differentiated adenocarcinoma infiltrating transmurally (H and E stain 10X).

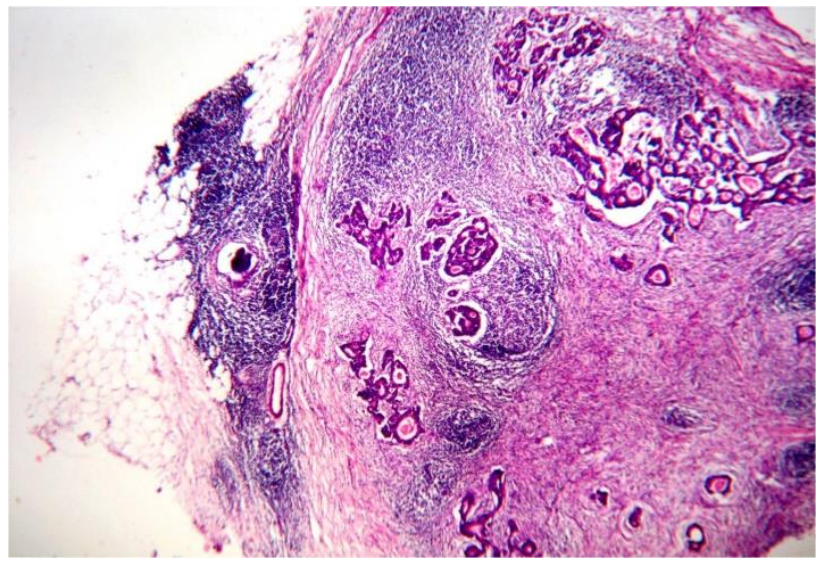

Figure 4: Lymph node section showing metastatic adenocarcinoma (H and E stain 10X). 
Grossly cut section of both ovaries revealed solid and cystic tumor mass with mucoid areas admixed with areas of hemorrhage and necrosis (Figure 1). Resected sigmoid colon revealed intraluminal polypoidal mass measuring $2 \times 2 \mathrm{~cm}$ (Figure 2). Multiple sections were taken from sigmoid colon mass, which revealed adenoma-carcinoma sequence with well differentiated adenocarcinoma infiltrating transmurally (Figure 3). Mucin secretions were seen only in focal areas. Single lymph node isolated in the mesenteric fat also shows tumor deposits (Figure 4) with spill over of tumor into surrounding adipose tissue. Multiple sections from both the ovarian masses also revealed well differentiated adenocarcinoma (Figure 5), with focal papillary projections and focal mucin secretions. The ovarian stroma showed dense desmoplastic response. In order to confirm the primary as ovarian or colonic, Immunohistochemistry with CK20 and CK7 was carried out. The CK20 was positive and CK7 was negative which confirmed the diagnosis as colonic primary with metastasis to bilateral ovaries. Hence final histopathological diagnosis of well differentiated adenocarcinoma of sigmoid colon with metastasis to bilateral ovaries/ Krukenberg tumor, stage pT3N1M1 was issued.

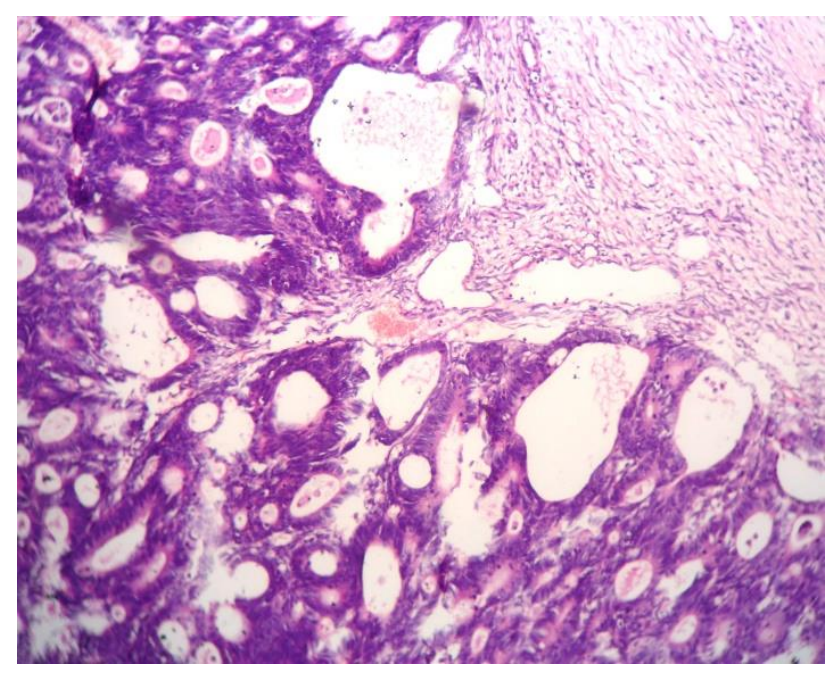

Figure 5: Sections from both the ovarian masses also revealed well differentiated adenocarcinoma with focal mucin secretions and desmoplastic ovarian stroma (H and E stain 20X).

Post-operative CT scan revealed focal lesion in the left lobe of liver and focal mesenteric fat with enhancing metastasis. Pelvic region and rest of peritoneal cavity did not show any evidence of metastasis or residual disease. Patient was posted for post-operative chemotherapy. She received Capecitabine $1 \mathrm{gm} / \mathrm{m}^{2}$ on day 1 to 14 and Injection Oxaliplatin $130 \mathrm{mg} / \mathrm{m}^{2}$ on day 1 , once in three weeks. She has received six cycles of chemotherapy and on eight month follow up she is free of disease. However, she has been advised for long time follow up in the future.

\section{DISCUSSION}

Over the last two decades, the urbanization and increasing modernization of India has transformed education, lifestyle, health-care access and longevity, and has contributed to an increased risk profile for chronic diseases such as cancer. ${ }^{1}$ Among these ovarian cancer is one of the commonest gynaecological cancers and ranks among the top five deadliest cancers in most countries. ${ }^{2}$ According to the center for disease control and prevention, as per the latest available data, the incidence of ovarian cancer for the year 2011 is around 15/100,000 women and death rate is around 9/100,000 women. This high rate of mortality warrants early diagnosis and treatment of the diseased individual. ${ }^{2}$

Tumors of the ovary are majority of the time primary with a small percentage of secondary malignancy. Metastasize to the ovaries can occur from many organs, including the stomach, small intestine, colon, rectum, gall bladder, appendix, pancreas, breast, uterus, fallopian tube, and peritoneum. Stomach is the primary site in most Krukenberg tumor cases (70\%). Carcinomas of colon, appendix, and breast are the next most common primary sites. ${ }^{2}$ These are referred to as Krukenberg tumor, accounting for $1 \%$ to $2 \%$ of all ovarian tumors. It was first described in 1896, by Friedrich Krukenberg, a German gynaecologist and pathologist, and he presumed it to be a new type of primary ovarian neoplasm. The criteria for diagnosis of Krukenberg tumor is: (1) cancer in the ovary, (2) the presence of mucin-producing neoplastic signet-ring cells, and (3) ovarian stromal sarcomatoid proliferation. $^{3}$ Recently, specific immunohistochemical methods have been tried in order to identify the site of primary neoplasm. ${ }^{4}$

Metastasis to the ovary spreads through various pathways. Direct spread or via other routes, like blood vessels, lymphatics, and surface implantation from intraabdominal cancers. ${ }^{5}$ Since the time of Krukenberg in 1896, all ovarian tumors which enlarge the ovaries uniformly without distortion, which present alterations of fibrous areas with mucinous-cell groups, and which propagate along the lymphatics are called "Krukenberg tumors". Having said so it is not that easy to diagnose a Krukenberg's tumor and it always needs a high level of suspicion. Literature has reported various atypical manifestations of Krukenberg tumor. ${ }^{6}$

Krukenberg tumor occurs with greatest frequency in women in the fourth and fifth decade of life. They are in most instances bilateral and produce a general enlargement of the ovary, which keeps its form and is usually free of adhesions. They are accompanied by ascitis; they metastasize early, and are almost invariably fatal.

Immunohistochemistry is very useful in differentiating primary versus secondary which is very essential to know outcome of the disease. Panel of IHC stains need to be 
carried out may include CK20, CK7, CA-125, HAM 56, and CEA. Positive staining for CEA and CK20 is typical of metastatic intestinal adenocarcinomas in the ovary. In contrast, although not specific, positive staining for CA$125, \mathrm{CK} 7$ and HAM 56 is much more typical of primary ovarian carcinomas.

In conclusion, Krukenberg's tumor is a rare bilateral ovarian metastatic tumor with primary usually from stomach. They are generally solid tumors and reveal signet ring cell adenocarcinoma on microscopy. Our case is interesting for the fact that, bilateral ovarian tumors were solid and cystic with very small primary tumor located in sigmoid colon. It did not reveal signet ring cells on microscopy, but showed well differentiated adenocarcinoma. The case report highlights the importance of preoperative workup needed to search for primary tumor, as Krukenberg's tumors are known for poor outcome.

Funding: No funding sources Conflict of interest: None declared Ethical approval: Not required

\section{REFERENCES}

1. Ghaffar A, Reddy KS, Singhi M. Burden of noncommunicable diseases in South Asia. British Med Journ. 2004:328:807-10.

2. Ferlay J, Shin HR, Bray F, Forman D, Mathers C, Parkin DM. Cancer Incidence and Mortality Worldwide. International Agency for Research on Cancer. 2010.

3. Novak C, Gray LA. Krukenberg tumor of the ovary: clinical and pathological study of four cases. Surgery Gynecology and Obstetrics. 1938:66;157-67.

4. Okamoto T, Matsumura N, Mandai M. Distinguishing primary from secondary mucinous ovarian tumors: an algorithm using the novel marker DPEP1. Modern Pathology. 2011;24:267-76.

5. Parker RT and Currier JL. Metastatic tumors of ovary. Gynecologic Oncology. 1992:987-1000.

6. Al-Agha M, Nicastri AD. An in-depth look at Krukenberg tumor: an overview. Archives of Pathology Laboratory Medicine. 2006:130:1725-30.

Cite this article as: Elamurugan S, Sinhasan SP, Sabita SC, Bupathy A. Ovarian primary or secondary: cracking the nut. Int J Reprod Contracept Obstet Gynecol 2016;5:3637-40. 